\title{
Using Social Media Networks to Conduct Questionnaire Based Research in Social Studies Case Study: Family Language Policy
}

\author{
Orly Kayam \\ Department of Language and Literacy Studies, Zinman College at the Wingate Institute, \\ Netanya, Israel \\ Email: orlyka@wincol.ac.il \\ Tijana Hirsch \\ Department of Language and Literacy Studies, Zinman College at the Wingate Institute, \\ Netanya Israel \\ Email: tijana-h@wincol.ac.il
}

Accepted: July 07, 2012 Published: July 31, 2012

Doi:10.5296/jsr.v3i2.2176 URL: http://dx.doi.org/10.5296/jsr.v3i2.2176

\begin{abstract}
The Internet has permeated all aspects of daily life in many areas of the world. Its advancement and feasibility affects how we work, socialize, and spend our time. The advent of social media networks has greatly contributed to these changes and has become the place where people on the Internet are. In light of this new social movement, we propose that social media networks can and should be viewed as the potential way of reaching participants in social studies research. In the current paper we review Internet's social effects research and provide an example of how the Internet and more specifically, the social media networks and other Internet tools can be utilized to perform a questionnaire based social research study in the area of Family Language Policy. We provide the 'how to' guide of conducting such research by sharing the technique employed in our study.
\end{abstract}

Keywords: Internet, research, social media networks, family language policy, social studies, questionnaire

\section{Introduction}

The Internet is a tool which connects people, makes it easier to maintain personal and social connections and also the connections to parts of oneself that have become less salient at a 
particular stage in life. In many economically developed societies, the use of computers and the Internet has become prevalent in the business/work and the private sectors. Majority of Americans, for example, have adopted the computer and Internet use for personal and economic reasons (Cummings \& Kraut, 2002). The advent of the information age and the accessibility of computers for personal use are present at the time when large portions of world's population have emigrated from their heritage lands into new countries, languages, and cultures, for limited or extended periods of time. The Internet with computers and other internet connected devices such as smart phones and tablets have become vehicles for communication maintenance between family members who live far away, and a tool for maintaining connections to heritage languages (HL) and heritage cultures among other things. New immigrants often cite and credit computer and Internet tools such as the e-mail, Skype, Facebook, and other Social Media tools, for easing their maintenance of contact with family member in their native countries and friends close and far. Considering this reality, it is almost natural to consider the Internet as a natural extension for communication between the researchers in the areas of social studies, as in our case dealing with questions about Family Language Policy (FLP), with their prospective participants. It is where the people spend their time and where they can be reached.

\subsection{Background}

Internet is extensively used for interpersonal communication today. It is used as a tool to stay in touch with distant relatives and cultures, encounter others from areas of the world we would otherwise not have a chance to communicate with, and to socialize and give and receive support. It is a tool which brings people together online, which in turn can affect our personal relationships, behaviors, and interactions offline. It was Hiltz and Turoff (1978) who pioneered the investigations concerned with the differences between computer-mediated communication's and other types of communication's influence on social interaction. More recently, Pollet, Roberts, \& Dunbar, (2011) investigated the relationship between the use of social network sites and instant messaging with offline social network size and quality or emotional closeness. They found that the large online social network size did not correspond to a larger offline network, nor did it correspond with emotionally closer offline relationships. Some researchers argue that online socializing replaces offline socializing, negatively affecting the quality of relationships between family members and offline friends (see Nie\& Hillygus, 2002). Others argue that online communication via social networks and instant messaging programs takes place between offline or existing friends, rather than new, online friends, therefore strengthening those relationships (Boyd \& Ellison, 2008). Valkenburg (2007), Boneva, Quinn, Kraut, et al. (2006) and Liu \& Larose (2008) among others have argued that social networking sites have a positive effect on wellbeing and are indeed effective in building high-quality relationships or friendships.

Internet is a medium of communication which encompasses and effects many different areas. It is to be examined in social, psychological, economical and other related terms and fields (O’Leary, Orlikowski, \& Yates, 2002). In terms of economics, work, and professional relationships, for example, “....although distributed work has existed for centuries, highly 
interdependent workgroups whose members are geographically distributed are a relatively recent phenomenon, made possible by improvement in computing and telecommunications, including the Internet. These new forms of working have caused researchers to reexamine how shared context and trust, often taken for granted in face-to-face settings, have their influence on group performance (Olson \& Olson, 2002; Rocco, 1998).”

In short, technological advancements and accessibility have affected how we work, how we socialize, how we spend our time, how we obtain information, how we make decisions, etc. Accordingly, the effects of these technological advancements are investigated throughout the research world, in many different areas of study, focusing on different aspects of the interaction between the technologies and individuals or groups. Although effects and relationships of communication and social networking over the internet are examined, only recently, have the researchers begun to use the Internet to perform research on topics not necessarily related to the Internet, but rather as the medium or a sort of cyber-laboratory for their experiments.

In this paper we will outline some advantages and disadvantages of the Internet as the cyber-laboratory in data collection using the questionnaire. We will also share our experiences of performing online research using Social Media channels in the area of Family Language Policy (FLP) using a questionnaire and the ways that we dealt with constraints or the disadvantages of the Internet as the tool for data collection. In doing so we will address different tools and social media platforms we used to create our questionnaire and to reach our participants respectively. This paper contributes to the area of social studies research by addressing the relatively new approaches and tools available in performing human-subjects research. It can be viewed as the 'how to' guide for researchers interested in conducting online research, and a starting point for all those considering this path.

\subsection{Advantages of Online Survey Research}

1. Cost - The Internet "lowers many of the costs associated with collecting data on human behavior...” (Kraut, Olson, M. Banaji, Bruckman, Cohen, \& Couper, 2003). In Internet research, facilities are not necessary. The personal computer and the Internet serve as the lab for research performance. With no need for facilities, maintenance of those facilities and utilities, as well as staff salaries; costs of data collection are substantially lowered. Surveys conducted in person or via the telephone require a person to supervise and carry out data collection. Online surveys are automated. The computer provides all the necessary tools and materials for successful data collection.

2. Time -Experimenter is able to perform experiments online yielding a collection of data from hundreds or thousands of participants with minimal intervention on part of the researchers (Nosek, Banaji, \& Greenwald, 2002a). Researchers and research assistants are able to maximize the use of their time, as the data collection is mostly left up to the Internet tool (see below) itself and the willingness of the participants. 
3. Participants -Research study can be posted on relevant websites, pages, newsletters, and distribution lists with the purpose of reaching potential research participants. The participant recruitment can be personalized, and it can target general or more focused communities (Couper, Traugott, \& Lamias, 2001). Samples can be specialized, large, or diverse depending on needs of the research project. They can, but do not have to be confined to a certain geographical area. For example, "researchers no longer need access to introductory psychology classes to recruit research subjects and often do not need grant money to pay them"(Kraut, Olson, M. Banaji, Bruckman, Cohen, \& Couper, 2003).

4. Voluntary Participation-Participants in the online studies have the benefit of the very real choice on whether to participate in the study or not. Unlike with the students in introductory social sciences courses, online participants will feel less pressure to please the researcher or the pressure to participate.

5. Human -Error -Although things can go wrong with computer-aided data collection, such errors are more likely to be documented. Human error is sometimes difficult to detect and/or document. For example, ensuring that same instructions are given to each and every participant prior to or following the survey is easily set up. The computer does not change the way that it delivers information to the participant. "With conventional, paper-based questionnaires, transcription of survey answers is an expensive and potentially error-prone process (p.6)" (Kraut, Olson, Banaji, Bruckman, Cohen, \& Couper, 2003). Today, there are many software packages, some free of charge, which collect the data in form of a database, and some even going further to provide statistical analyses on the data collected.

\subsection{Disadvantages of Online Survey Research}

1. Representativeness of the Data and Random Sampling - Although the Internet has become prevalent in many countries around the world, not everyone is on the Internet. Those who use the Internet and choose to participate in the studies do differ from those unreachable via the Internet. Clearly, this is a constraint of research findings performed on the Internet and should be made clear to those who are reading the results. This, however, is also the case with an in-person data collection procedure whose participants come from the introductory social sciences university courses. Sampling in Internet research studies is also not randomized. Participants are reached via certain channels which make them a part of a specific (online or social) group. 
2. Data Pollution - The possibility of ill intent of some people has to be considered. This could include multiple submissions by the same person, for example. Furthermore, some researchers site the possibility of lack of commitment on part of the participant, in that he or she may spend less time and invest less energy in his/her participation.

3. Protection of Human Subjects - The Internet is a large place with many ways of connecting information and identifying people. Although some view it as one of the major disadvantages of Internet research, in online surveys, we found this to be minimally problematic. (for more information see below in section "FLP Online Survey Study”) (Kraut, Olson, Banaji, Bruckman, Cohen, \& Couper, 2003) Informed consent and debriefing are also of concern; however, we found this not to be a problem (as will be discussed above).

\section{The Case Study: Family Language Policy Survey via Social Media Sources}

In the current paper we focus on online social studies research using surveys or questionnaires, it is important to mention that Internet research is a tool which can be utilized in many more ways. Research via the Internet can be set up to perform complex psychological and social experiments, tracking participant behaviors, response times, observations of social behaviors, and others. In the above sections of this work we have described some of the advantages and disadvantages regarding the online research studies using questionnaires or surveys and have outlined relevant literature review on topics encompassed by this work. We will now discuss our experiences with an online research study using a questionnaire and describe in detail the tools utilized and steps taken in our research.

\subsection{Study and Target Population}

Our study was an online-based FLP study focusing on attitudes and choices of parents of young children belonging to a minority language group, within a larger and dominant language context in Israel. Our target population were immigrant parents of young children (preschool age) from English speaking countries in Israel.

\subsection{Why Online?}

The reason for approaching our target population via the Internet is twofold: convenience and inclusion. We wanted to make it as convenient as possible for participants to take part in our study. Parents of young children are very busy and asking them to take time out of their busy days, physically, would have made it impossible for some parents to participate. Our suspicion that parents would be more easily reached via the Internet and more specifically the social media platforms was supported by a recent finding which says that parents, particularly mothers, spend more time on Facebook after giving birth 
(Bartholomew, Schoppe-Sullivan, Glassman, Kamp Dush, and Sullivan, 2012). By making the questionnaire more easily accessible to our target population across Israel it was possible for us to obtain a sample which is representative of the immigrant group regardless of their place of residence in Israel. Our aim was to reach this linguistic community regardless of their location within the country, and regardless of their immigration status or religious orientation. We wanted to gain an insight into FLP processes within the linguistic community at large: new immigrants, expats, those living in Israel for a very long time, religious, non-religious, Zionist or not, Jewish, non-Jewish, etc. We recognized the Internet as a tool which would make this goal possible. Furthermore, by reaching different corners of the country during the same time frame, we removed the temporal distortion from our data. All of the participants completed the questionnaire during the same 3 months and during the same season.

\subsection{How?}

We created the survey using the free of charge Google Documents tool (docs.google.com). The first page of the survey included an explanation of the purpose of the study, provided contact and background information on the researchers and the institution conducting the research, assured participants of the anonymity of the study, and gave participants an option to contact the researchers with additional questions, comments, and/or if they were interested in taking part in future sections of the research. Essentially, with upfront communication of the study's nature, lack of risks or penalties for choosing not to participate in the study, as well as possibility to contact researchers directly, our aim was to create a safe and anonymous environment where participants could share their experiences and attitudes at no potential risk. The option to e-mail researchers in order to participate in future sections of the study was in no way connected to the questionnaire. The participants had to voluntarily and through their own prerogative open their e-mail browsers and send an e-mail to the researchers, making it impossible to link their e-mail addresses to the answers provided on the questionnaire.

The questionnaire itself consisted of general demographic questions and FLP practices, experiences, and attitudes. The participant's answers were recorded automatically into a spreadsheet associated with Google Documents. Participants were reached via different channels (discussed below) and had to click on the link of the study in order to enter the questionnaire. No identifying information was collected.

\subsection{About the tool: Google Documents}

Google Documents (docs.google.com) make it possible to create a survey, document, or a presentation which is kept on the server and is easily accessed from anywhere where there is an Internet connection. The security level of the document or the survey created can be adjusted according to need and can be shared with collaborators and/or participants as desired. The 
sharing and viewing options range from having the document freely viewable by anyone on the Internet, by those who have the link to the document, through sign-in only, to private and un-viewable by others, as needed. The questionnaire or the document created in Google Documents is editable by only those people who are given permissions by the owner of the document. This tool is particularly attractive as it is available online, is not affiliated with any website, and does not require login information. Creating surveys on Facebook, in contrast, requires participants to log into the survey, therefore divulging all the information available on participant's Facebook page. Those without a Facebook account cannot participate in the survey without creating a Facebook account, whereas, in Google Documents anyone invited or with a link to the questionnaire can access it and participate without divulging any identifying information. Once participants complete the questionnaire and click on the submit button, data is stored in the Google Docs spreadsheet. This spreadsheet is safe and once again only reachable or viewable by those with assigned permissions by the document's owner. Once data collection is complete, the questionnaire can be closed to further participation. The data file can easily be converted into a spreadsheet (among other options), which can then be uploaded into the data analysis tool such as SPSS. Another interesting feature is the "Summary of Responses" which provides a visual representation of the responses provided in the form of pie chart views. We found this free tool to be very user friendly and more than adequate for carrying out our study. For larger studies, one may consider a paid survey tool such as those listed on the Social Psychology Organization website.

\subsection{Informed Consent and Debriefing}

All the information about the study, its purpose, and the information about researchers and the institution were presented as the first page of the survey. Since this study did not include deception, there was no need for additional debriefing after the completion of the study. All the information was given upfront, including the contact information of the researchers for those interested in learning about the results of the study, comments, questions, and/or interest in future studies.

\subsection{Reaching Participants}

In order to reach participants of our particular linguistic minority group in Israel, we researched different channels. We made a list of all (to our knowledge) of the social organizations, volunteer organizations, community pages, Facebook groups, community centers, religious organizations, and city offices created for assistance and absorption of our target linguistic minority group across Israel. We contacted these channels via e-mails and/or phone calls and requested their help in sharing the link of our study on their websites, newsletters, magazines, e-mail lists, forums, Facebook pages, Twitter, and LinkedIn pages. Most organizations and individuals were happy to help, some more than others, and the link to our study began to circulate among different groups of the community at large. We also joined and posted the link to our study on all (to our knowledge) Facebook pages representing different parts of our linguistic community. The researchers joined different social groups on Facebook, either by 
requesting permission from group's moderator (if the group was closed) or by adding the group to their Facebook page (if the groups were 'open'). We shared the information and the link to our study on these pages, requesting from all those who fit into our category and who are interested to participate to do so. We also shared the information and the link of the study on Facebook pages which could not be joined but rather served as pages representing different organizations and/or business catering to our target population. In all cases, we simply posted the information on the "Wall" of different social groups on Facebook. Our link was then visible to anyone who was linked to or visited that specific social group's page. Although the information on our study was posted on Twitter and LinkedIn accounts of different organizations, as well as our own Twitter and LinkedIn pages, we found Facebook to be the most effective in 'spreading the word.' We were actively able to reach out to different community and social groups of English speakers in Israel and at times the community members responded by clicking 'like' on our post or posting a comment in response to our post. We also received numerous e-mails in which participants mentioned reaching our study's link via (more often than not) one of the Facebook pages. Again, although survey research conducted online cannot be viewed as representative of the community at large, we aimed to make it as accessible and representative as possible of different sectors of the community present online.

\subsection{Safety \& Honesty}

In our opinion, by safeguarding participant's anonymity and safety in communicating with out potential consequences we raise their comfort levels and encourage honest and unedited responses to research questions. It is therefore of utmost importance to keep the data collection procedures safe and discreet. The topic of our research, as mentioned above, was the FLP of English speaking immigrants in Israel. Language and immigrant experiences are very personal and sharing of one's true experiences, feelings, practices, and attitudes can only be done without potential loss of anonymity. In her book, Viv Edwards (2004) explains: Language equals Culture: The native language is tied into peoples' history, identity, and roots. Since our study focused on language and immigrant experiences within the family, our study was asking for a glimpse into not only the identity of our participants, but also the personal and private experiences and attitudes within the family and the larger immigrant context. Protecting the participant's status of anonymity was of utmost importance.

\section{Response Rates: Results of our approach}

Our experience with using social media networks to conduct questionnaire based research is very positive. We were able to reach participants who would otherwise be unreachable simply because of the distance and the amount of effort and time it would take on their part to participate in our study. The tools available online were not only free of charge, but also very user friendly. In this study, we used the tools of today to reach our participants where they are today: online, on social media networks. 


\section{MlMacrothink}

Journal of Sociological Research

ISSN 1948-5468

2012, Vol. 3, No. 2

The study circulated on different online channels (described in detail above) for 3 months (April, May, June). In that time, 232 participants completed the questionnaire. Considering the fact that response rates to online surveys are very low coupled with the wide distribution of our subjects, we found this number to be a great success. As hoped, we managed to reach a wide audience, across the country of Israel, with participants residing in all corners of the country. Immigrants from North, Center, South, and East, villages, settlements, and cities such as Jerusalem, Tel Aviv, and Haifa were reached and participated in our research. At the end of the questionnaire an open area for comments was provided and as mentioned earlier we provided researchers' contact information for any comments and/or questions. Two comments pertaining to the participants' experiences with the study format as e-mailed to us unprompted are included in the Appendix A.

During the period of data collection, researchers ensured that the link to the study appeared on different online channels, answered participants' questions and continued reviewing pertinent literature on the subject of the investigation.

During the course of the study, out of the 232 participants 66 participants sent comments and expressed their interested in learning about the results and/or participating in future sections of the research.

\section{Conclusion}

Internet is a communication tool that has, in many areas of the world, permeated all facets of daily life, from social aspects to professional, individuals, families, and societies at large. It is a coffer of experiences, attitudes, behaviors, causes, and effects in itself which is and should be examined from many different perspectives and using different methodologies. It is also a naturally occurring cyber-laboratory, a place where people are and can be reached in order to shed light on different topics, perspectives, experiences, attitudes, etc. and where they may be more willing to spare their time and offer their input. The development of Internet based tools such as the social media networks has brought people together, taking them out of geographically distinct areas into groups where borders, names of countries or cities, and temporal and distance related obstacles are of no issue. On the Internet, in the social media networks, people can get together no matter how near or far.

Our experience with performing questionnaire based attitude study on FLP via the Internet and more specifically the social media has been positive. We found people to be responsive and eager to participate. The tools available on the Internet today, as outlined above, can be thought of as performing research in the way that is environmentally friendly and cost effective: by removing the need to financially support physical space or a lab where participants would complete their surveys, travel costs associated with reaching the lab on both part of the researcher and participants were removed, instead of papers computer screens were used, participants could complete the survey at the time that best suited them, and risks of researcher related inconsistencies were removed. In a short period of time we were able to 
reach and obtain responses from participants across a large geographical area, without offering payment or incentives other than their contribution to the research community at large. We are positive, that in this study, our participants took part voluntarily, without a shadow of a doubt. We protected their anonymity, approached them where they spent their time (the Internet) and the resulting response was positive. We speculate that because we brought relevant research to the relevant forums the outcome yielded real interest and desire to participate in our study.

References

Boneva BS, Quinn A, Kraut RE, et al. (2006) Teenage communication in the instant messaging era. In Kraut R., Brynin M., Kiesler S., eds. Computers, phones, and the Internet: Domesticating information technology. New York: Oxford University Press.

Boyd DM, Ellison NB. (2008) Social network sites: Definition, history and scholarship. Journal of Computer-Mediated Communication, 13, 210-38.

Couper, M. P., Traugott, M. W., \& Lamias, M. J. (2001). Web survey design and administration. The Public Opinion Quarterly, 65(2), 230-253.

Cummings, J. N., \& Kraut, R. (2002). Domesticating computers and the Internet. The Information Society, 18(3), 1-18.

Edwards, V. (2004) Multilingualism in English-Speaking World. Blackwell Publishing Ltd.

Hiltz, S., \& Turoff, M. (1978). The network nation: Human communication via computer. Cabridge, MA: MIT Press.

Kraut, R., Olson, J. S., Banjai, M. R., Bruckman, A., Cohen, J., \& Couper, M. Psychological Research Online: Opportunities and Challenges. American Psychological Association.

Liu X, Larose R. (2008) Does using the Internet make people more satisfied with their lives? The effects of the Internet on college students' school life satisfaction. Cyber Psychology \& Behavior, 11, 310-20.

Nie NH, Hillygus DS. (2002)Where does Internet time come from?A reconnaissance. IT \& Society 1, 1-20.

Nosek, B. A., Banjai, M. R., \& Greenwald, A. G. (2002). E-research: Ethics, security, design, and control in psychological research on the Internet. Journal of Social Issues, 58(1), 161-176.

O'Leary, M., Orlikowski, W., \& Yates, J. (2002). Distributed work over the centuries: Trust and control in the Hudson's Bay Company, 1670-1826. In P. Hinds \& S. Kiesler (Eds.), Distributed Work (pp. 27-54). Cambridge MA: MIT Press. 
Olson, G. M. \& Olson, J. S. (2000). Distance Matters. Human-Computer Interaction, 15(2-3), 139-178.

Rocco., E. (Ed.). (1998). Trust breaks down in electronic contexts but can be repaired by some initial face-to-face contact. Los Angeles, California, United States: ACM Press.

Valkenburg P, Peter J. (2007). Online communication and adolescent well-being: Testing the stimulation versus the displacement hypothesis. Journal of Computer-Mediated Communication 12, 1169-82. 